\title{
Multi-product economic lot scheduling problem with manufacturing and remanufacturing using a basic period policy
}

\author{
Simone Zanoni, Anders Segerstedt, Ou Tang and Laura Mazzoldi
}

\section{Linköping University Post Print}

\section{Tweet}

N.B.: When citing this work, cite the original article.

Original Publication:

Simone Zanoni, Anders Segerstedt, Ou Tang and Laura Mazzoldi, Multi-product economic lot scheduling problem with manufacturing and remanufacturing using a basic period policy, 2012, Computers \&amp; industrial engineering, (62), 4, 1025-1033.

http://dx.doi.org/10.1016/j.cie.2011.12.030

Copyright: Elsevier http://www.elsevier.com/

Postprint available at: Linköping University Electronic Press

http://urn.kb.se/resolve?urn=urn:nbn:se:liu:diva-77534 


\title{
Multi-product economic lot scheduling problem with manufacturing and remanufacturing using a basic period policy
}

\author{
Simone Zanoni ${ }^{\mathrm{a}}$, Anders Segerstedt ${ }^{\mathrm{b}, \mathrm{c}}$, Ou Tang ${ }^{\mathrm{d}}$, Laura Mazzoldi ${ }^{\mathrm{a}}$ \\ ${ }^{a}$ Department of Mechanical and Industrial Engineering, University of Brescia, via Branze 38, 25123 \\ Brescia, Italy \\ ${ }^{\mathrm{b}}$ Industrial Logistics, Luleå University of Technology, SE-971 87 Luleå, Sweden \\ ${ }^{c}$ IDBK, Narvik University College, N-9505 Narvik, Norway \\ ${ }^{\mathrm{d}}$ Division of Production Economics, Department of Management and Engineering, Linköping \\ University, SE-581 83 Linköping, Sweden
}

\begin{abstract}
In this research we study the multi-product economic lot scheduling problem (ELSP) with manufacturing and remanufacturing opportunities. Manufacturing and remanufacturing operations are performed on the same production line. Both manufactured and remanufactured products have the same

quality thus fulfil the same demand stream. Tang and Teunter (2006) firstly studied this type of economic lot scheduling problem with returns (ELSPR) and presented a complex algorithm for the optimal solution. More recently Teunter et al (2009) proposed several heuristics to deal with the same problem using more computational efficient approaches. However, both studies have limited the attention to the common cycle policy with the assumption that single (re)manufacturing lot is used for each item in each cycle. Relaxing the constraint of common cycle time and a single (re)manufacturing lot for each item in each cycle, we propose a simple, easy to implement algorithm, based on Segerstedt (1999), to solve the model using a basic period policy. Several numerical examples show the applicability of the algorithm and the cost savings.
\end{abstract}

Keywords: ELSP, Returns, Remanufacturing, Reverse logistics

\section{Introduction}

The economic lot scheduling problem (ELSP) refers to the challenge of accommodating several items to be produced on a single machine in a cyclical pattern, with a goal of minimizing the total set-up and 
holding costs. This ELSP was first proposed by Rogers (1958), who identified the need of joining the ELQ (economic lot quantity) problem of different items, with the aim of scheduling them on single production centre in order to minimize the total set-up and holding costs. During the past decades a significant amount of research has been reported on this problem, with various directions of extensions. Bomberger (1966) introduced the upper and lower bounds for the total cost, together with a dynamic programming procedure, in order to find a feasible scheduling solution, illustrated with examples which are widely used in later ELSP studies. Doll and Whybark (1973) relaxed the common cycle policy and introduced a model with different production frequencies for different items, named basic period (BP) policy; they also proposed an iterative procedure to find the near optimal frequencies, resulting in a reduction of total cost.

Elmaghraby (1978) provided a comprehensive review for the ELSP problem. Davis (1990) proposed a mixed integer programming (MIP) formulation for the ELSP, which afterwards was enhanced by Cooke et al (2004). Nevertheless a procedure to find an optimal feasible solution for the general version of the problem is not known yet, while it is clear that it is NP-hard problem since Hsu (1983) who also showed that the NP-hardness increases with the capacity utilization ratio. Given its difficulties, researchers approached the problem using analytic solutions to find simplified versions of the problem, or adopted heuristic methods for the original one. Dobson (1987) showed that the timevarying lot size approach often produced a feasible schedule, as well as giving a better solution quality than the basic period approach, although its computation complexity should also increase substantially. Recently Raza and Akgunduz (2008) presented a Simulated Annealing algorithm considering the timevarying lot size approach and presented a comparative study of heuristic algorithms on ELSP.

A complementary challenging problem is to extend the ELSP model, which involves only manufacturing operations, to the ELSPR model, which involves also remanufacturing operations on returned items, performing both manufacturing as well as remanufacturing activities on a single production line.

Figure 1 represents the system components involved in a ELSPR model and the main relationships among them. There is a single resource (production line) that provides manufacturing and remanufacturing operations of some items, which are then sent to the serviceable stock, and finally to the market in order to satisfy the demand $(d)$. After they enter the market, a certain amount of returns $(\beta d)$ comes back to the production facility in order to be remanufactured, and a recoverable stock is available to collect them. 


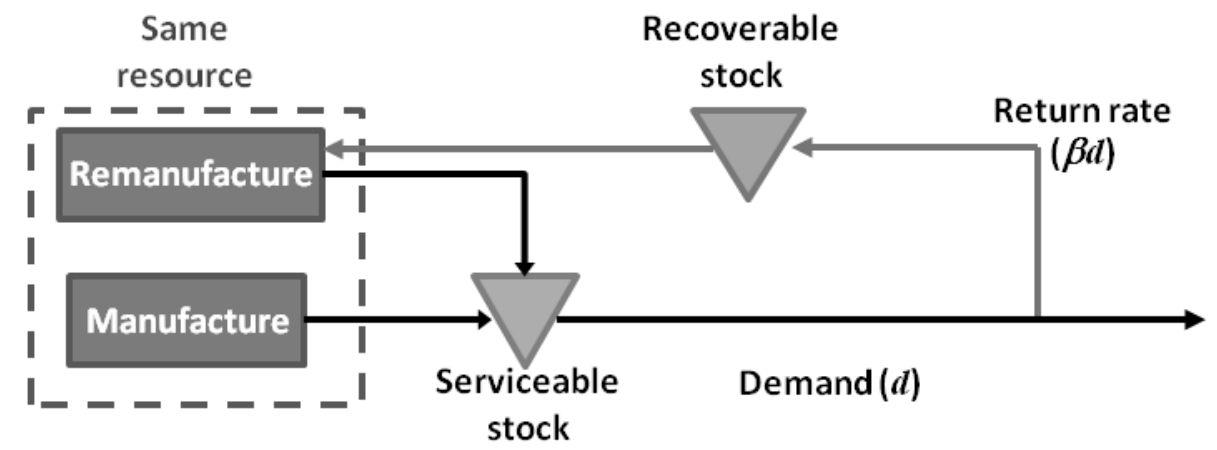

Figure 1 - ELSPR system.

The serviceable and recoverable inventories' profiles for two items, with a common cycle policy (that is a single manufacturing lot followed by a single remanufacturing lot) are reported in Figure 2. During serviceable stock filling from manufacturing process, the returns are collected into the recoverable stock. This recoverable stock increases till remanufacturing lot starts.

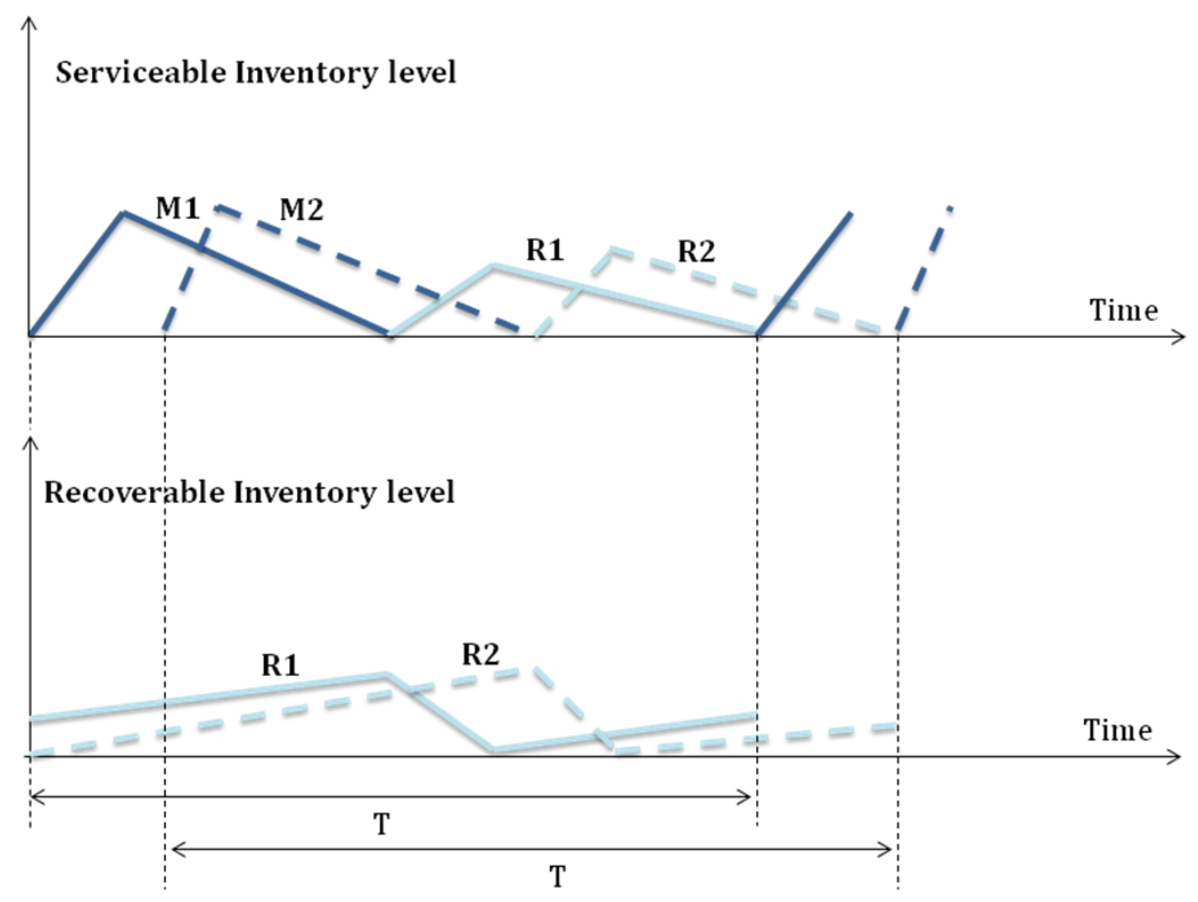

Figure 2 - Serviceable and recoverable inventory patterns for ELSPR system with two items under a common cycle policy ( $\mathrm{M}=$ Manufacturing, $\mathrm{R}=$ Remanufacturing).

This Economic Lot Scheduling Problem with Returns (ELSPR) was first introduced by Tang and Teunter (2006). They studied a specific case of a company which was involved in car parts 
manufacturing for service markets. The items produced were new items as well as remanufactured returned products. Moreover manufacturing as well as remanufacturing operations for all items were performed on the same production line, according to the ELSP base model.

The ELSPR problem becomes rather complicated compared with the classic ELSP model even under the common cycle assumption. This is due to the fact that two stock points (recoverable and serviceable) need to be managed; in addition the serviceable stock is pooling items from manufacturing and remanufacturing processes. In the ELSPR, not only the lot size, but also the production schedule influences the inventory level, while for the traditional ELSP, any schedule leads to the same stock level patterns and average stock levels. The relevance of scheduling for the ELSPR makes it a more complex problem than the ELSP.

Tang and Teunter (2006) firstly developed an algorithm for the ELSPR with the formulation of a mixed integer linear program (MIP). Their study is based on common cycle policy and the solution method combines the search for the optimal value of the cycle time and schedules for all items. They further reminded that MIP formulation is rather complex to perform, and its programming is quite tedious; further the algorithm iteration procedure may be too time consuming, depending on the problem size.

By dropping the exact problem formulation, Teunter et al (2009) developed heuristics to enhance the solution efficiency. They generated 120 sets of data to test their heuristics' performance. We also refer to these 120 sets of data in this paper, with the same purpose. Teunter et al (2008) further presented the ELSPR considering 2 separate production lines for manufacturing and remanufacturing item lots. The dedicated production lines for manufacturing and remanufacturing bring a significant reduction in holding costs and an increase in scheduling flexibility.

This paper further studies the above mentioned ELSPR problem by extending the scheduling policy from the common cycle to a basic period policy. Our aim is to introduce a simple scheduling policy which can be solved with near-optimal solutions, and which has a great potential to improve the cost performance in the system. More specifically, by introducing approximations in the cost function, we develop a heuristic to reduce the computational complexity, compared with exact solution method in the common cycle approach in Tang and Teunter (2006). The cost saving is then investigated in the numerical examples.

The rest of the paper is organized as follows. In the next section we propose an algorithm, based on a variation of Segerstedt (1999), that provides a basic period solution approach for the ELSPR problem. 
In section 3 we provide some numerical results, based on the 120 sets of data introduced by Teunter et al (2009), to test our algorithm performance. Finally we advance some conclusions and possible future developments.

\section{The algorithm}

As mentioned before, in both Tang and Teunter (2006) and Teunter et al (2009), common cycle policy is used to face the ELSPR. Even if the demand is small or the set-up time is long for one particular item, it has to be manufactured and remanufactured once during the common cycle. This policy obviously simplifies the scheduling complexity, but on the other hand it might increase the total cost in the system. Thus, in this paper our aim is to relax the common cycle assumption, considering less restricted scheduling options, so as to reduce the total costs.

The scheduling complexity of ELSPR models is mainly due to the co-existence of manufacturing and remanufacturing operations, making the modelling expression of serviceable inventory holding cost quite complicated, especially when adopting the basic period (BP) policy: in fact the serviceable inventory level of an item depends on the schedule coordination of manufacturing and remanufacturing operations on that item, and this scheduling complexity is greater for ELSPR models than for ELSP models (as we declare in the Introduction section). This is the reason why we propose to manage a ELSPR model involving $\mathrm{N}$ items as an adjusted ELSP model involving $2 \cdot \mathrm{N}$ items. In order to do this we also propose to manage the serviceable inventory as 2 separate inventories, one for manufactured items, and one for remanufactured items, presenting what we named "Independently managed serviceable inventory" (IM), which differs from the traditional management approach, named "Jointly managed serviceable inventory" (JM). This disconnection will of course increase the serviceable inventory holding costs, but this assumption allows us to obtain a closer form expression for scheduling problem adopting various production frequencies, related to the implementation of the basic period policy. The solving algorithm is then adapted from the one proposed by Segerstedt (1999). The heuristic consists of an iterative procedure and the main idea is to find a balance between the replenishment and the inventory holding costs. Similarly to the economic order quantity model (EOQ), it can be experienced that as the ratios between the replenishment and the inventory holding costs for each installation are closer to one the solution becomes better. This main idea has also been applied to other problems with high-quality results: the Joint Replenishment Problem (Nilsson et al, 2007) and the One Warehouse N-retailer Problem (Abdul-Jalbar et al, 2010). 


\subsection{Assumptions and notation}

Assumptions follow the conventions in the traditional ELSP formulation, with some additional concerns for returns:

1. only one item can be manufactured/remanufactured at a time on the single production resource;

2. demand rate, return proportion, manufacturing rate and remanufacturing rate are deterministic and constant;

3. manufacturing and remanufacturing set-up times and costs are independent of production sequence;

4. inventory holding costs are directly proportional to inventory levels for serviceable and recoverable inventories;

5. backlogs or lost sales are not allowed.

The following notation is used throughout the paper:

$N \quad$ number of items (each item can be either manufactured or remanufactured)

$d_{i} \quad$ demand rate in units per unit time (production days) for item $i$

$\beta_{i} \quad$ return proportion of item $i$

$p_{i}^{m / r}$ number of units of item $i$ manufactured/remanufactured during one time unit

$s_{i}^{m / r}$ set-up time, time units per manufacturing/remanufacturing lot for item $i$

$h_{i}{ }^{r} \quad$ inventory holding cost for recoverable inventory per unit and time unit of item $i$

$h_{i}{ }^{s} \quad$ inventory holding cost for serviceable inventory per unit and time unit of item $i$

$A_{i}{ }^{m / r}$ set-up cost for manufacturing/remanufacturing lot for item $i$

$T$ Common cycle time length (in time units) during which all items should be produced at least once

W Basic period length (in time units)

$f_{i}^{m / r}$ number of times (frequency) that manufactured/remanufactured item $i$ is produced during a Common cycle time 
The superscript $\mathrm{m} / \mathrm{r}$ means that the parameter is referred to manufactured products $(m)$ or to remanufactured $(r)$ products.

It has to be noted that even though a common cycle time $T$ is introduced in the above notation, in the policy described in this paper items can be produced several times within a common cycle time $T$, therefore $T$ is an integer number of times $W\left(\right.$ where $\left.W=T / \max _{i}\left\{f_{i}^{m / r}\right\}\right)$.

\subsection{Serviceable inventory: Manufactured and Remanufactured items}

In this section we present expressions for serviceable inventory. First we consider the case of jointly managed serviceable inventory (JM), i.e. which means that manufactured lots as well as remanufactured lots are held together in a single serviceable inventory (same as the common cycle policy in Tang and Teunter,2006). Then we introduce independently managed serviceable inventory (IM), where the manufactured and remanufactured lots are disconnected and each lot has to meet just a portion of the demand $d_{i}$ for the i-th item. In particular serviceable inventory filled by the manufactured lots of the $\mathrm{i}$-th item has to satisfy $\left(1-\beta_{i}\right) \cdot d_{i}$ demand, while the serviceable filled by the remanufactured lots of the i-th item has to satisfy $\beta_{i} \cdot d_{i}$ demand. The main purpose of introducing IM policy is to simplify the expression for serviceable inventory, without the need of considering the scheduling constraints introduced by Tang and Teunter (2006).

Adopting JM approach, the total serviceable inventory related to the i-th item will be empty before a manufacturing or remanufacturing lot of the same item starts, while adopting IM approach the manufactured and remanufactured serviceable inventories are independently managed and they reach zero at different times: manufacturing related serviceable inventory for the i-th item reaches zero when the production of a manufactured lot of the i-th item starts, while the remanufacturing related serviceable inventory for the i-th item reaches zero when the production of a remanufactured lot of the i-th item starts.

Even though the adoption of IM approach introduces additional serviceable inventory holding costs, compared to the JM approach, it allows an easier formulation and solution for the ELSPR model under a Basic Period (BP) policy.

\subsubsection{Jointly managed serviceable inventory}


Considering a serviceable inventory where manufactured and remanufactured lots of a specific item $i$ are stocked together, we can divide the total cycle time $T$ into 2 separate time periods: a first "manufacturing" time period, followed by a "remanufacturing" one. Considering an ideal case with the minimal total serviceable inventory holding cost, manufacturing or remanufacturing operations on item $i$ should start when the serviceable inventory of item $i$ reaches zero (Figure 3 ). In this circumstance, (1$\left.\beta_{i}\right) T$ and $\beta_{i} T$ represent the time periods during which demand $d_{i}$ is satisfied by the manufactured lot and by the remanufactured lot, respectively, of item $i$.

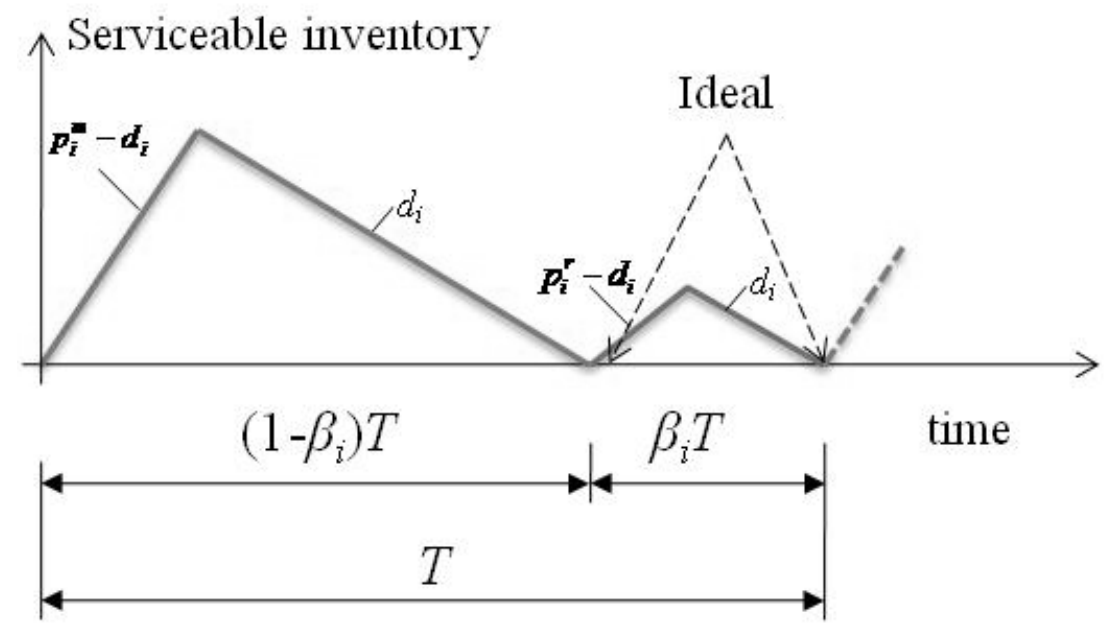

Figure 3 - Jointly managed serviceable inventory levels.

As indicated by Tang and Teunter (2006), the above illustrated ideal case may not always occur since dealing with a multi-item system, involving the production of all lots (manufactured and remanufactured) on the same line, the schedule of one lot should be limited by the coordination between remanufacturing and manufacturing operations of other lots. Thus, apart from the ideal serviceable inventory holding cost, additional holding cost for serviceable should be added to the nonideal case, as reported in Tang and Teunter (2006).

Average Serviceable Inventory $(\mathrm{JM})=A C_{\text {Ideal }}+A C_{\text {Additional }}$

$$
\begin{aligned}
& =\left(1-\beta_{i}\right) \frac{\left(1-\beta_{i}\right) T d_{i}}{2}\left(1-\frac{d_{i}}{p_{i}^{m}}\right)+\beta_{i} \frac{\beta_{i} T d_{i}}{2}\left(1-\frac{d_{i}}{p_{i}^{r}}\right) \\
& +d_{i}\left(\left(1-\beta_{i}\right)\left[f\left(x_{i}^{r}-x_{i}^{m}\right)-T\left(1-\beta_{i}\right)\right]^{+}+\beta_{i}\left[f\left(x_{i}^{r}-x_{i}^{m}\right)-T\left(1-\beta_{i}\right)\right]^{-}\right)
\end{aligned}
$$

In the above reported expression, the modelling complexity is mainly associated with $x_{i}^{r}$ and $x_{i}^{m}$ which indicate the starting points of remanufactured and manufactured lots of item $i$, respectively. 


\subsubsection{Independently managed serviceable inventory}

In this case the return proportion $\beta_{i}$ is used to split the demand $d_{i}$ into "manufacturing demand" and "remanufacturing demand", $\left(1-\beta_{i}\right) d_{i}$ and $\beta_{i} d_{i}$, respectively, for item $i$. It can be noted that, in such a configuration, the total serviceable inventory level doesn't usually reach zero, as the manufacturing or remanufacturing of a new lot can start independently from the serviceable stock level. The advantage of IM is that the manufacturing and remanufacturing schedules can be managed independently and thus it reduces the modelling complexity. Thus the $N$ items ELSPR model can be reduced to a $2 \cdot N$ items ELSP model. The average serviceable inventory level for the independently managed serviceable inventory becomes

Average Serviceable Inventory $(\mathrm{IM})=\frac{T\left(1-\beta_{i}\right) d_{i}}{2}\left(1-\frac{\left(1-\beta_{i}\right) d_{i}}{p_{i}^{m}}\right)+\frac{T \beta_{i} d_{i}}{2}\left(1-\frac{\beta_{i} d_{i}}{p_{i}^{r}}\right)$

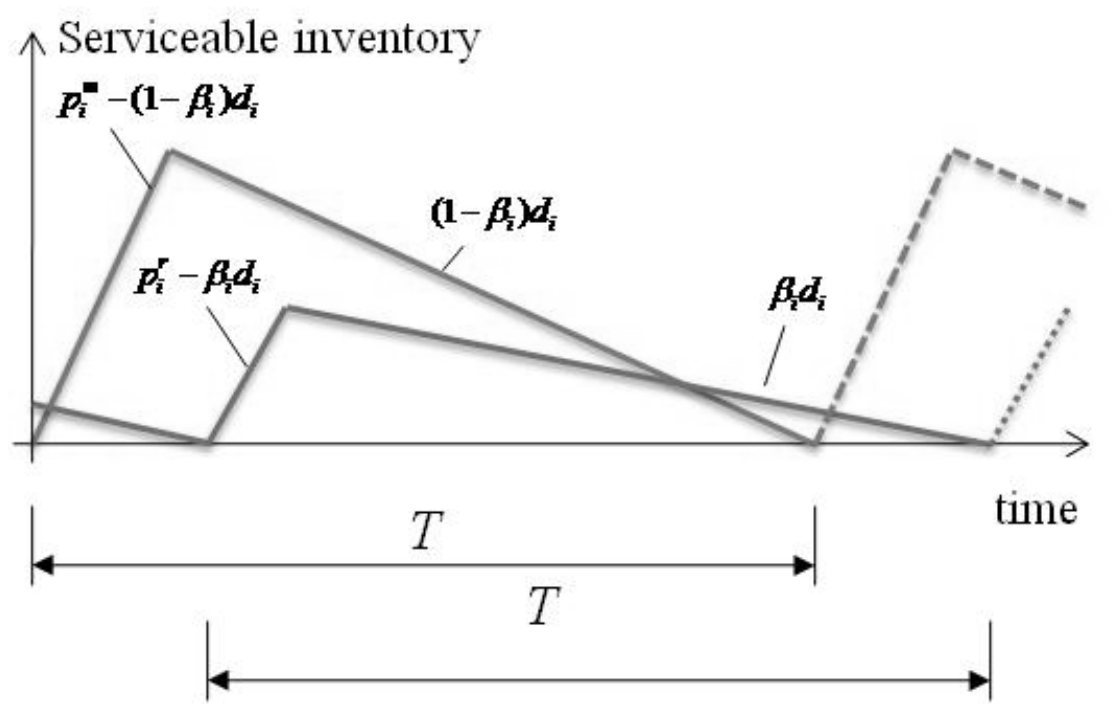

Figure 4 -Independently managed serviceable inventory levels.

\subsubsection{Serviceable inventory increase (IM vs $J M$ )}

Comparing JM and IM, the latter one should increase the serviceable inventory holding cost since it disconnects the coordination of the two production lots (manufactured and remanufactured). If we ignore the additional cost, and compare only the ideal cost related to JM with the cost related to IM, the difference can be written as: 
$\frac{\operatorname{Avg} \operatorname{Serv} \operatorname{Inv}(\mathrm{IM})-\operatorname{Avg} \operatorname{Serv} \operatorname{Inv}(\mathrm{JM})}{\operatorname{Avg} \operatorname{Serv} \operatorname{Inv}(\mathrm{JM})}=\frac{2 \cdot\left(\beta_{i}-\beta_{i}^{2}\right)}{\left(1-\beta_{i}\right)^{2}\left(1-\frac{d_{i}}{p_{i}^{m}}\right)+\beta_{i}^{2}\left(1-\frac{d_{i}}{p_{i}^{r}}\right)}$

It should be noted that formula (3) presents the maximum increase since only the ideal serviceable inventory holding cost is used as a reference in the jointly managed situations. Formula (3) can also graphically presented, as illustrated in Figure 5, with a varying coefficient $\beta_{i}$ and other parameters constant. It is illustrated that the highest error occurs when a return proportion $\beta_{i}$ equals to $50 \%$.

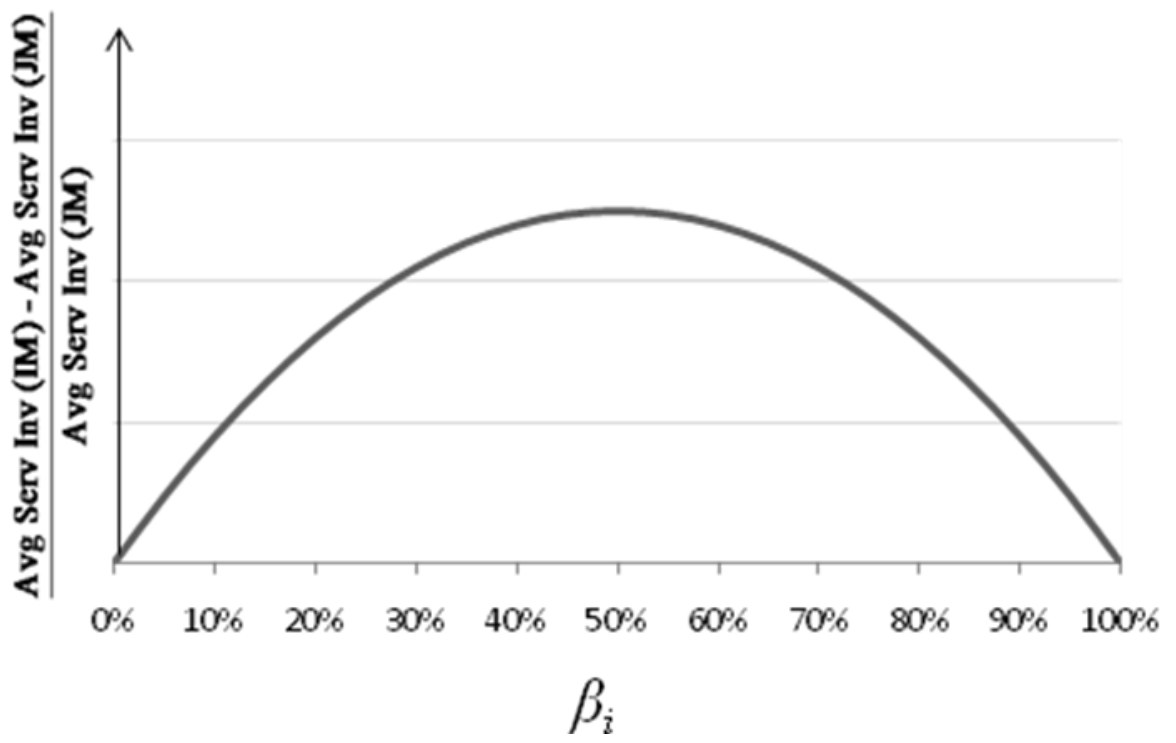

Figure 5 - Maximum cost increase related to coefficients $\beta_{i}$

\subsection{The BP policy algorithm}

With the independently managed serviceable inventory (IM), we obtain a simple expression for serviceable inventory holding cost. The following proposed algorithm refers to the approximated model, and it is based on a variation of the one in Segerstedt (1999), which considers a Basic Period (BP) policy, and involves the power-of-two heuristics to find items' production frequencies. We would like to remind that using power-of-two heuristics generates a further approximation error which never exceeds 6\% (Roundy, 1989).

\subsubsection{Description of algorithm's steps}

First of all it has been necessary to set all items frequencies to a power-of-two desired value, and to compute $T_{\min }$ value; then the model can be solved considering the common cycle policy: as a result the "approximated common cycle cost", which is the reference for the next algorithm steps, is computed. 


\section{Step 0}

Set $p=0$

Set $T_{\max }$

$T_{\max }$ is the maximum allowed value of cycle length $T$, which can be chosen as the maximum number of time units during the production campaign.

\section{Step 1}

Set $\quad f_{i, p}^{m / r}=2^{Z} \forall i(i=1 . . N)$

where $2^{Z}$ is the maximum power-of-two frequency with which the items can be either manufactured or remanufactured during a common cycle (where $Z$ is a nonnegative integer number). A big $Z$ often leads to a long computation time in the algorithm but it also improves the opportunity to reach a lower cost (when the algorithm explores BP solutions).

The entire set of frequencies for all items (manufactured $(m)$ and remanufactured $(r)$ ) can be written as a vector:

$F_{p}=\left\{f_{i, p}^{m / r}\right\}$

\section{Step 2}

Compute $T_{\text {min,p }}$, which is the minimum allowed value to accommodate production (and set-up) of all items during the cycle time.

There is a strong constraint related to $T_{\text {min,p }}$ : should the optimal value of cycle time $T_{p}$ be lower than $T_{\min , p}$ the capacity utilization of the manufacturing/remanufacturing resource will be greater than $100 \%$, which is an impossible situation.

So we compute $T_{\min , p}$ as:

$$
T_{\min , p}=\frac{\sum_{i=1}^{N} f_{i, p}^{m} \cdot s_{i}^{m}+\sum_{i=1}^{N} f_{i, p}^{r} \cdot s_{i}^{r}}{1-\left(\sum_{i=1}^{N}\left(1-\beta_{i}\right) \cdot d_{i} / p_{i}^{m}+\sum_{i=1}^{N} \beta_{i} d_{i} / p_{i}^{r}\right)}
$$

$$
\text { If } T_{\min , p}<0 \text { or } T_{\min , p}>T_{\max } \quad \mathrm{STOP}
$$

\section{Step 3}


Then, compute the lowest cost according to a possible specified time interval $T_{p}$ (so that $T_{p} \geq T_{\min , p}$ and

$$
\begin{aligned}
& \left.T_{p} \leq T_{\max }\right): \\
& \begin{aligned}
C_{p}= & \sum_{i=1}^{N} \frac{f_{i, p}^{m} \cdot A_{i}^{m}}{T_{p}}+\sum_{i=1}^{N} \frac{f_{i, p}^{r} \cdot A_{i}^{r}}{T_{p}}+h_{i}^{s} \cdot \sum_{i=1}^{N} T_{p} \cdot\left(1-\beta_{i}\right) \cdot \frac{d_{i}}{2} \cdot\left(1-\frac{\left(1-\beta_{i}\right) \cdot d_{i}}{p_{i}^{m}}\right) \cdot \frac{1}{f_{i, p}^{m}}+ \\
& +\left(h_{i}^{s}+h_{i}^{r}\right) \cdot \sum_{i=1}^{N} T_{p} \cdot \beta_{i} \cdot \frac{d_{i}}{2} \cdot\left(1-\frac{\beta_{i} \cdot d_{i}}{p_{i}^{r}}\right) \cdot \frac{1}{f_{i, p}^{r}}
\end{aligned}
\end{aligned}
$$

\section{Step 4}

After evaluating common cycle cost, which involves all frequencies equal to a fixed power-of-two value, we compute the cost of the basic period alternatives, with a different set of frequencies that balance the set-up and holding costs.

Compute:

$$
\left\{\begin{array}{c}
\text { ratio }_{i, p}^{m}=\frac{\frac{f_{i, p}^{m} \cdot A_{i}^{m}}{T_{0}}}{h_{i}^{s} \cdot T_{0} \cdot\left(1-\beta_{i}\right) \cdot \frac{d_{i}}{2} \cdot\left(1-\frac{\left(1-\beta_{i}\right) \cdot d_{i}}{p_{i}^{m}}\right) \cdot \frac{1}{f_{i, p}^{m}}} \\
\operatorname{ratio}_{i, p}^{r}=\frac{\frac{f_{i, p}^{r} \cdot A_{i}^{r}}{T_{0}}}{\left(h_{i}^{s}+h_{i}^{r}\right) \cdot T_{0} \cdot \beta_{i} \cdot \frac{d_{i}}{2} \cdot\left(1-\frac{\beta_{i} \cdot d_{i}}{p_{i}^{r}}\right) \cdot \frac{1}{f_{i, p}^{r}}}
\end{array} \quad \forall=1 . . N\right.
$$

Where ratio $_{i, p}^{m / r}$ represents the ratio between set-up costs and holding costs for a considered item $i$ (manufactured or remanufactured) at step $p$ : it can be observed that similarly to the basic EOQ model, the closer ratio $o_{i, p}^{m / r}$ approaching to 1 , the better balanced are set-up and holding costs, thus the lower is the total cost.

\section{Step 5}

Set $p=p+1$

We calculate the set of frequencies ensuring that the set of frequencies comply with the power-of-two assumption. 
$f_{i, p}^{m}=2^{\left.\log _{2}\left(\mid T_{0} \sqrt{\frac{h_{i}^{s} \cdot\left(1-\beta_{i}\right) \cdot \frac{d_{i}}{2} \cdot\left(1-\frac{\left(1-\beta_{i}\right) \cdot d_{i}}{p_{i}^{m}}\right)}{A_{i}^{m}}}\right)+0.5\right] \mid} \quad \forall \quad i=1 . . N$

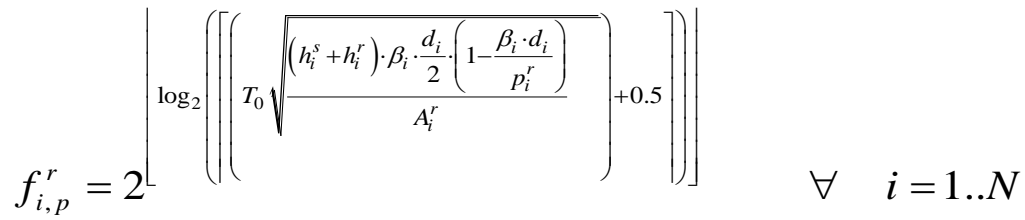

If $f_{i, p}^{m / r}<1$

Then $f_{i, p}^{m / r}=1$

If $f_{i, p}^{m / r} r>2^{Z}$

Then $f_{i, p}^{m / r}=2^{\mathrm{Z}}$

\section{Step 6}

Compute again $T_{m i n, p}$ according to (4).

Then, compute the lowest cost according to a possible specified time interval and the chosen set of frequencies $F_{p}$ :

$C_{p}=\min _{T_{p}}\left(\begin{array}{c}\sum_{i=1}^{N} \frac{f_{i, p}^{m} \cdot A_{i}^{m}}{T_{p}}+\sum_{i=1}^{N} \frac{f_{i, p}^{r} \cdot A_{i}^{r}}{T_{p}}+h_{i}^{s} \cdot \sum_{i=1}^{N} T_{p} \cdot\left(1-\beta_{i}\right) \cdot \frac{d_{i}}{2} \cdot\left(1-\frac{\left(1-\beta_{i}\right) \cdot d_{i}}{p_{i}^{m}}\right) \cdot \frac{1}{f_{i, p}^{m}}+ \\ +\left(h_{i}^{s}+h_{i}^{r}\right) \cdot \sum_{i=1}^{N} T_{p} \cdot \beta_{i} \cdot \frac{d_{i}}{2} \cdot\left(1-\frac{\beta_{i} \cdot d_{i}}{p_{i}^{r}}\right) \cdot \frac{1}{f_{i, p}^{r}}\end{array}\right)$

If $\quad C_{p}<C_{p-1}$

Then $\quad C_{\text {best }}=C_{p} ; \quad F_{\text {best }}=F_{p}$

Else $\quad C_{\text {best }}=C_{p-1} ; \quad F_{\text {best }}=F_{p-1}$

So as to show the application of the algorithm's steps hereafter it is applied to a small numerical example. Data for the numerical example (arbitrarily generated), limited to three items either manufactured or remanufactured, are reported in Table 1.

\begin{tabular}{|c|r|r|r|}
\hline $\boldsymbol{i}$ & \multicolumn{1}{|c|}{$\mathbf{1}$} & \multicolumn{1}{|c|}{$\mathbf{2}$} & \multicolumn{1}{c|}{$\mathbf{3}$} \\
\hline$\beta_{i}$ & 0.07 & 0.79 & 0.47 \\
\hline$d_{i}$ & 188 & 4 & 284 \\
\hline
\end{tabular}




\begin{tabular}{|c|r|r|r|}
\hline$p_{i}{ }^{m}$ & 9416 & 11571 & 2181 \\
\hline$p_{i}{ }^{r}$ & 27647 & 1349 & 3374 \\
\hline$s_{i}{ }^{m}$ & 0.047 & 0.033 & 0.126 \\
\hline$s_{i}{ }^{r}$ & 0.033 & 0.082 & 0.041 \\
\hline$A_{i}{ }^{m}$ & 6 & 2 & 2 \\
\hline$A_{i}{ }^{r}$ & 24 & 4 & 8 \\
\hline$h_{i}{ }^{r}$ & $1.24 \cdot 10^{-5}$ & $6.83 \cdot 10^{-4}$ & $1.13 \cdot 10^{-3}$ \\
\hline$h_{i}{ }^{r}$ & $5.00 \cdot 10^{-7}$ & $2.40 \cdot 10^{-4}$ & $4.23 \cdot 10^{-4}$ \\
\hline
\end{tabular}

Table 1 - Numerical example data for the algorithm's step application

\section{Step 1}

$p=0$

$T_{\max }=24 * 30=720$ hours

$T_{\max }$ has been chosen arbitrarily so as to be equal to the amount of hours available in 1 month (30 days), 24 hours/day, and giving the significance of hours to the time unit for the values reported in Table 1.

$\mathrm{Z}=4$, so $2^{\mathrm{Z}}=16$ which is the maximum allowed production frequency for all items

Then: $F_{0}{ }^{m}=\{16 ; 16 ; 16\} ; F_{0}{ }^{r}=\{16 ; 16 ; 16\}$

\section{Step 2}

$T_{\min , 0}=6.6577$

\section{Step 3}

$C_{0}=5.7780 ; T_{0}=254.7590$

\section{Step 4}

Then ratio $_{i, 0}^{m}=\{22.2453 ; 27.5146 ; 0.0998\}$ and ratio $_{i, 0}^{r}=\{1115.7919 ; 10.8502 ; 0.3172\}$

\section{Step 5}

$p=1$

which is $F_{1}^{m}=\{2 ; 2 ; 16\}$ and $F_{1}^{r}=\{1 ; 4 ; 16\}$

\section{Step 6}

$T_{\min , 1}=3.6703$

$C_{l}=3.2577$

$T_{1}=132.6083$ 
$C_{1}<C_{0}$ then:

$C_{\text {best }}=C_{1}=3.2577$

$F_{\text {best }}=F_{1}$ and $F_{\text {best }}{ }^{m}=\{2 ; 2 ; 16\}$ and $F_{\text {best }}{ }^{r}=\{1 ; 4 ; 16\}$

\subsubsection{The BP approach: BP feasibility test}

Once the best solution is found, it is necessary to check its feasibility. This is an issue which has been discussed extensively for the traditional ELSP model. For example, Nilsson and Segerstedt (2008) suggested that if the sum of the set-up and production times of all item, with different frequencies, is less than a common cycle time $(T)$, this is both a necessary and sufficient condition for feasibility, but a new replenishment may have to start before the inventory reaches zero. In a different way, Doll and Whybark (1973) proposed the following definition: "To be feasible, a production schedule must not have more than one item scheduled at the same time and the lot for each item must be sufficiently large to meet the demand for $T$, where $T$ is a multiple of a basic period."

Here we intend "feasibility" of a BP result if it is possible to adjust the items with a schedule that encompasses frequencies $f_{i}^{m / r}$ which are:

- $\quad$ at least one $f_{i}^{m / r}$ equals to 1 ;

- at least one $f_{i}^{m / r}$ is different from all other frequencies in $F_{p}$;

and the sum of the set-up times and production times of all item scheduled in each basic period, must be less than or equal to the basic period $(W)$.

We would remind here that if an item is (re)manufactured more than once, its production lot is split into $f_{i}^{m / r}$ small lots, in order to satisfy, anyway, its demand during the cycle time $T$.

We propose 3 different BP feasibility tests, ranging from an easy to implement but very restrictive one (sufficient but not necessary criteria), to a "point-to-point" feasibility checking for the solution found, which requires a more complex computational procedure, but ensures the feasibility (necessary and sufficient criteria). The intermediate test is as fast and easy to implement as the first one, but it is not so restrictive (thus necessary but not sufficient criteria).

We introduce the first "BP feasibility test" in order to determine which instances are certainly "BP feasible", so as to reduce the number of instances checked by the "point-to-point" method, which is time consuming. With the same purpose, we implement the second BP feasibility test to determine which instances are certainly "NON-BP feasible". So, excluding "sure BP feasible" and "sure NON-BP 
feasible", we implement "point-to-point" feasibility checking only for those instances which result is "false" for the two first "BP feasibility tests".

\section{$\underline{\text { Proposition }}$}

Let $S_{o p t}$ be the best solution found for a given dataset $D S$, and $\sigma_{i}^{m / r}$ the total manufacturing/remanufacturing time which includes production and set-up times for one lot of item $i$.

During the common cycle $T$ the item $i$ can be produced $f_{i}^{m / r}$ times, so that $\sigma_{i}^{m / r}$ can be computed as follows:

$$
\left\{\begin{array}{l}
\sigma_{i}^{m}=\left(\frac{\left(1-\beta_{i}\right) \cdot d_{i} / p_{i}^{m}}{f_{i}^{m}}\right) \cdot T+s_{i}^{m} \\
\sigma_{i}^{r}=\left(\frac{\beta_{i} \cdot d_{i} / p_{i}^{r}}{f_{i}^{r}}\right) \cdot T+s_{i}^{r}
\end{array}\right.
$$

BP feasibility test 1

$$
\sum_{i=1}^{N}\left(\sigma_{i}^{m}+\sigma_{i}^{r}\right) \leq W
$$

The first proposed BP feasibility test represents a sufficient but not necessary criterion, which enables us to discard instances a priori; this obviously lets us identify instances that are certainly BP feasible solutions, but it has the disadvantage of "losing possible feasible instances", because of the restrictiveness of such a criterion. Looking at (11) it is possible to ensure that $S_{\text {opt }}$ is a feasible solution if all item lots could be produced (computed as the sum of all production and set-up time for all item lots) during the basic period $W$.

As it can be easily understand, implementing such a restrictive criterion, we realize that very little number of instances could be BP feasible. So we modify the used criterion and we choose a new one as in (12).

\section{$\underline{\text { BP feasibility test } 2}$}

Let $I_{k}$ denote the set of items with the maximum frequency (i.e. $\max \left\{f_{i}^{m / r}\right\}$ ) than:

$$
\sum_{i \in I_{k}} \sigma_{i}^{m / r}+\max _{i}\left\{\sigma_{i}^{m / r}\right\} \leq W
$$


The second test is less restrictive than the first one and it is satisfied if the sum of operation time of the item with the maximum frequency plus the maximum operation time can be adjusted in the basic period time.

Unlike the previous criterion, this one doesn't represent a sufficient condition on which it could be possible to declare the feasibility of a specific solution found, but, on the contrary it is possible to declare the "NON-feasibility" of the same solution. In fact, if the sum of production and set-up times of those item lots which are required to be outperformed in each time interval (each of value $W$ ) and the maximum production and set-up time computed exceeds the value $W$, it is possible to say that the basic period constraint is not respected, and such an instance can be declared "BP NON-feasible".

The next step is to build a schedule so as to guarantee "BP feasibility" for those instances which result is "false" for both the previous presented tests.

\section{BP feasibility test 3}

The scheduling rules used to allocate each production lot to a time interval (the basic period $W$ ) are following reported, and they are inspired by Tunasar and Rajgopal (1996):

1. Sort all frequencies $f_{i}^{m / r}$ in descending order, those items with the same frequencies $f_{i}^{m / r}$ are further sorted in a descending order of their $\sigma_{i}^{m / r}$.

2. Assign item $i$ at the top of the list to the $w$-th basic period slot (each basic period length is $W$, and the total number of basic period is $\max \left\{f_{i}^{m / r}\right\}=T / W$ ) which idle time value is the maximum among all time intervals. If item $i$ is assigned to the $w$-th basic period slot, it is also assigned in a similar fashion to the subsequent $f_{i}^{m / r}$ basic period slots with intervening gaps of $(\varepsilon-1)$ slots (where $\left.\varepsilon=\left(\max \left\{f_{i}^{m / r}\right\}\right) / f_{i}^{m / r}\right)$, which means that the time required by the lots assigned to the $w$-th time slot exceeds the $W$ value. If such an assignment cannot be completed go to Step 4.

3. Remove the item from the list and update the idle time in each $w$-th basic period slot. If the list is non-empty return to Step 2; otherwise a BP feasible assignment has been made and the algorithm stops.

4. The algorithm cannot find a BP feasible assignment.

\subsection{The modified BP policy}

If the feasibility check proposed according to the three procedures above reported fails, we have to modify the schedule of the items so as to look for alternative schedule to the common cycle one. In 
fact, for all instances not "BP-feasible" the common cycle schedule (i.e. each (re)manufacturing frequency, $f_{i}^{m / r}$, equals to 1 ), even with the proposed Independently Managed inventory, is always possible to be adopted.

The main problem that could occur when a production schedule is not "BP feasible" is that a backlog could arise during the cycle time $T$ for one or more items, in one or more basic period time slots. In order to quantify such backlogs, and with the purpose of avoiding their occurrence, we introduce a new procedure, based on the established set of (re)manufacturing frequencies $\left\{f_{i}^{m / r}\right\}$ and schedule generated from the BP feasibility test 3, using a two-step procedure.

\section{$\underline{\text { Step } 1}$}

Based on Leven and Segerstedt (2007), we propose a modification of the BP solution: the main idea is to compress the idle time at the end of the common cycle $T$, while in a traditional BP policy each $w$-th BP includes a certain amount of idle time. By introducing the "modified BP" we would like to generate a sequence of manufacturing and remanufacturing production batches without any idle time between them (starting producing an item as soon as the production of another item is finished). However modifying the production schedule in such a way the BP nature of the solution is violated and therefore it is necessary to calculate total cost of the modified schedule: in order to do it, it is necessary to calculate the holding cost according to the amount of items stocked either in the serviceable and in the recoverable inventories, during the time exactly they are stocked there. This modification of idle time allocation implies a "time interval variability", because BP slots starting and finishing times are no more a constraint for the beginning and the ending of set-up and production times, and so a time interval could be shorter or longer than the traditional BP slot (the total idle time is allocated in the last BP slot), however the number of time intervals is still $\max \left\{f_{i}^{m / r}\right\}$. More in detail the BP requires to schedule set-up and production times of an item during the same basic period slot and the total idle time is split into $w$ BP slots, where $w=\max \left\{f_{i}^{m / r}\right\}$, while adopting a modified BP policy the idle time is concentrated just before the end of the cycle time $T$. Moreover considering the BP policy when an item is (re)manufactured more than once during the cycle time $T$, the different item lots must be assigned to different basic period slots, one lot for each time slot, while adjusting production as proposed in "modified" BP policy means that, as set-up and production time of a new item lot starts as soon as the previous production lot is over, it may lead to the case with more than one lot of a certain item in the same time slot. 
So as to help to understand the effect of the "modified BP policy" in Figure 6 and Figure 7 are respectively represented the $\mathrm{BP}$ schedule and the modified BP schedule generated from it, referred to an arbitrary example.

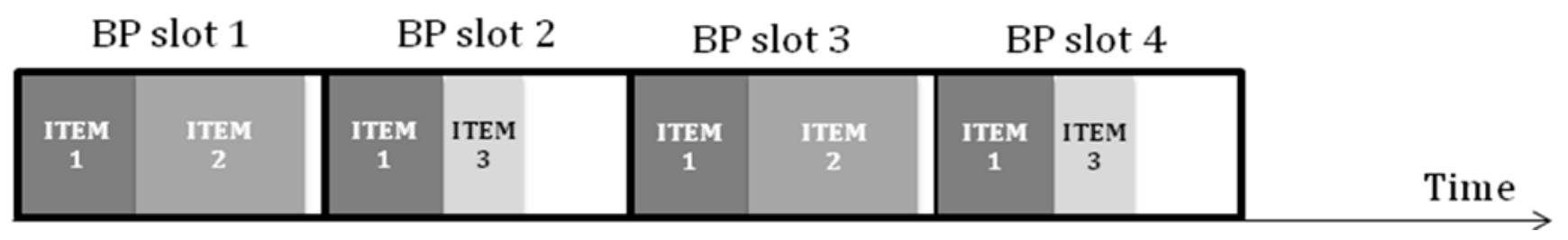

Figure 6-BP approach

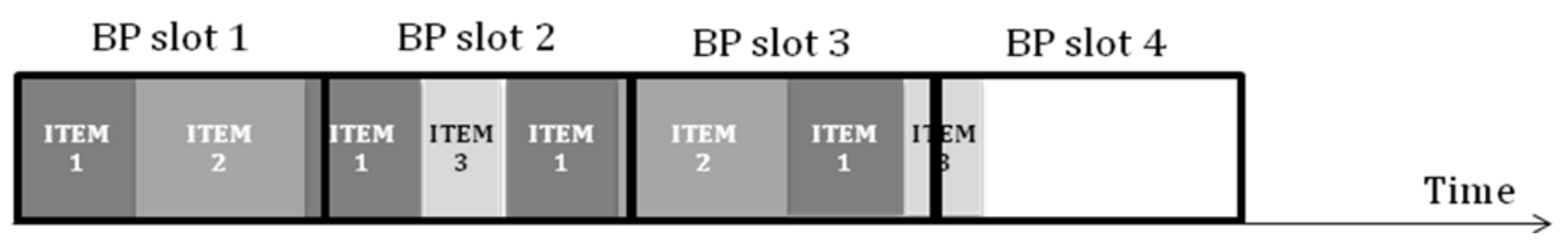

Figure 7 - Modified BP approach

\section{$\underline{\text { Step } 2}$}

If the first adjustment of the schedule is not enough to ensure the feasibility, it is possible to introduce an additional enhancement to the modified BP schedule, considering a non-zero inventory level, for those items that experience a shortage during the cycle time $T$ : the amount of such a initial stock level is exactly the quantity of shortage that arise considering a zero initial inventory level (this schedule adjustment is mainly based on Nilsson and Segerstedt (2008)). The main advantage in implementing this second step is to reach the feasibility for all instances (also for those we declared "BP NONfeasible"), at the cost of increasing holding costs for all items in the schedule (not only for those that experience a shortage.)

\section{Numerical study}

As mentioned before Teunter et al (2009) proposed a set of heuristics to solve ELSPR with the common cycle policy. In order to present some results of their proposed approach, they provided 120 sets of data (instances), generated from the famous Bomberger (1966) original lot sizing problem data, which has been often used by researches as a benchmark.

In this paper we refer to the same 120 instances, so as to compare our model performances with the results presented in Teunter et al (2009). Teunter et al (2009) grouped the 120 instances into 4 groups 
based on the capacity utilization (excluding set-up times) as follows: capacity utilization for instances 1 to 30 is less than $50 \%$; for instances 31 to 60 capacity utilization is between $50 \%$ and $75 \%$; for instance 61 to 90 capacity utilization is between $75 \%$ and $100 \%$; capacity utilization for instances $91-120$ is greater than $87 \%$.

In the following, we have implemented the BP policy, setting the maximum allowed frequency value equal to 64 (that corresponds to $Z=6$ ) in the numerical examples reported. A high frequency value may improve cost savings but it also increases computational time. Moreover a high frequency could be impractically to be implemented in scheduling.

As illustrative examples, Table 2 and 3 provide the parameters setting for instances 61 and 101 respectively, with a capacity utilization corresponding to $75 \%$ and $95 \%$.

\begin{tabular}{|c|r|c|r|c|c|c|r|r|r|c|}
\hline$i$ & \multicolumn{1}{|c|}{$A_{i}{ }^{m}$} & $s_{i}{ }^{m}$ & $A_{i}{ }^{r}$ & $s_{i}{ }^{r}$ & $h_{i}{ }^{s}$ & $h_{i}{ }^{r}$ & \multicolumn{1}{c|}{$p_{i}{ }^{m}$} & \multicolumn{1}{c|}{$p_{i}^{r}$} & \multicolumn{1}{c|}{$d_{i}$} & $\beta_{i}$ \\
\hline 1 & 23 & 0.066 & 48 & 0.002 & $3.700 \cdot 10^{-6}$ & $8.000 \cdot 10^{-7}$ & 29403 & 7735 & 171 & 0.93 \\
\hline 2 & 6 & 0.067 & 16 & 0.038 & $4.190 \cdot 10^{-5}$ & $1.320 \cdot 10^{-5}$ & 21405 & 5124 & 119 & 0.48 \\
\hline 3 & 3 & 0.280 & 13 & 0.159 & $4.610 \cdot 10^{-5}$ & $7.900 \cdot 10^{-6}$ & 10025 & 6519 & 323 & 0.28 \\
\hline 4 & 5 & 0.023 & 1 & 0.235 & $4.400 \cdot 10^{-5}$ & $1.250 \cdot 10^{-5}$ & 7809 & 4902 & 440 & 0.20 \\
\hline 5 & 101 & 0.906 & 30 & 0.757 & $2.167 \cdot 10^{-3}$ & $8.500 \cdot 10^{-5}$ & 145 & 106 & 36 & 0.03 \\
\hline 6 & 37 & 0.226 & 139 & 0.158 & $6.190 \cdot 10^{-5}$ & $5.310 \cdot 10^{-5}$ & 16050 & 4083 & 4 & 0.50 \\
\hline 7 & 333 & 1.776 & 573 & 1.272 & $9.310 \cdot 10^{-5}$ & $3.630 \cdot 10^{-5}$ & 2520 & 676 & 6 & 0.41 \\
\hline 8 & 31 & 0.432 & 273 & 0.587 & $4.356 \cdot 10^{-4}$ & $5.707 \cdot 10^{-4}$ & 1288 & 373 & 104 & 0.96 \\
\hline 9 & 304 & 0.384 & 454 & 1.149 & $1.733 \cdot 10^{-4}$ & $3.780 \cdot 10^{-5}$ & 377 & 932 & 56 & 0.96 \\
\hline 10 & 4 & 0.128 & 1 & 0.219 & $2.990 \cdot 10^{-5}$ & $2.300 \cdot 10^{-6}$ & 9113 & 5500 & 148 & 0.68 \\
\hline
\end{tabular}

Table 2 - Parameters setting for instance 61 .

\begin{tabular}{|c|r|r|r|c|c|c|r|r|r|r|}
\hline$i$ & $A_{i}{ }^{m}$ & \multicolumn{1}{c}{$s_{i}{ }^{m}$} & \multicolumn{1}{c}{$A_{i}{ }^{r}$} & $s_{i}{ }^{r}$ & $h_{i}{ }^{s}$ & $h_{i}{ }^{r}$ & $p_{i}{ }^{m}$ & \multicolumn{1}{c|}{$p_{i}^{r}$} & \multicolumn{1}{c|}{$d_{i}$} & \multicolumn{1}{c|}{$\beta_{i}$} \\
\hline 1 & 13 & 0.096 & 24 & 0.075 & $2.750 \cdot 10^{-5}$ & $1.700 \cdot 10^{-6}$ & 9476 & 20224 & 163 & 0.55 \\
\hline 2 & 1 & 0.046 & 5 & 0.078 & $9.097 \cdot 10^{-4}$ & $1.912 \cdot 10^{-4}$ & 9254 & 4132 & 8 & 0.61 \\
\hline 3 & 1 & 0.224 & 8 & 0.124 & $5.294 \cdot 10^{-3}$ & $2.790 \cdot 10^{-5}$ & 2225 & 1537 & 252 & 0.95 \\
\hline 4 & 2 & 0.008 & 5 & 0.107 & $1.572 \cdot 10^{-4}$ & $5.690 \cdot 10^{-5}$ & 6940 & 3923 & 195 & 0.92 \\
\hline 5 & 5 & 0.296 & 56 & 0.172 & $1.703 \cdot 10^{-4}$ & $1.790 \cdot 10^{-5}$ & 658 & 118 & 49 & 0.87 \\
\hline 6 & 11 & 0.083 & 71 & 0.012 & $2.590 \cdot 10^{-3}$ & $1.492 \cdot 10^{-3}$ & 9819 & 2275 & 8 & 0.75 \\
\hline 7 & 148 & 0.43 & 52 & 0.875 & $1.040 \cdot 10^{-5}$ & $1.096 \cdot 10^{-4}$ & 1967 & 492 & 20 & 0.61 \\
\hline 8 & 14 & 0.372 & 74 & 0.460 & $1.410 \cdot 10^{-5}$ & $1.600 \cdot 10^{-6}$ & 308 & 64 & 28 & 0.41 \\
\hline 9 & 52 & 0.309 & 218 & 0.066 & $3.490 \cdot 10^{-5}$ & $8.100 \cdot 10^{-6}$ & 468 & 317 & 29 & 0.18 \\
\hline 10 & 1 & 0.037 & 2 & 0.059 & $9.990 \cdot 10^{-5}$ & $2.900 \cdot 10^{-5}$ & 4901 & 3741 & 81 & 0.9 \\
\hline
\end{tabular}

Table 3 - Parameters setting for instance 101.

Table 4 reports the results of the proposed modified BP IM compared with the CC JM. While adopting the IM approach and using a modified BP policy for instance 61 generates a cost saving of $32.2 \%$ (compared to CC JM) and for instance 101 the modified BP IM leads to a cost saving (compared to CC JM) of $39.9 \%$. 


\begin{tabular}{|c|c|c|c|c|}
\hline \multirow{2}{*}{ Instance } & \multirow{2}{*}{$\mathrm{CU}(\%)$} & \multicolumn{2}{|c|}{ Total Costs } & $\begin{array}{c}\text { Total Costs } \\
\text { Deviation [\%] }\end{array}$ \\
\cline { 3 - 5 } & & CC JM & Modified BP IM & $\begin{array}{c}\text { Modified BP } \\
\text { IM } \\
\text { vs } \\
\end{array}$ \\
& & & & CC JM \\
\hline 61 & 75 & 28.32 & 19.21 & $-32.2 \%$ \\
\hline 101 & 95 & 51.22 & 30.77 & $-39.9 \%$ \\
\hline
\end{tabular}

Table 4 - Results for instances 61 and 101.

Then we examine all instances reported in Teunter et al (2009) and present the results of adopting modified Basic Period Independently Managed (Modified BP IM) and Commons Cycle Jointly Managed (CCJM) policies in Table 5.

\begin{tabular}{|c|c|c|c|c|}
\hline Instances & $\begin{array}{c}\text { Capacity } \\
\text { Utilisation }\end{array}$ & $\begin{array}{c}\text { Avg Total Costs } \\
\text { Deviation [\%] } \\
\text { Modified BP IM } \\
\text { vs CC JM }\end{array}$ & $\begin{array}{c}\text { \% of instances } \\
\text { with Total Cost } \\
\text { savings with } \\
\text { Modified BP IM } \\
\text { vs CC JM }\end{array}$ & $\begin{array}{c}\text { Max } \\
\text { Total Costs } \\
\text { Deviation [\%] } \\
\text { Modified BP IM } \\
\text { vs CC JM }\end{array}$ \\
\hline $1-30$ & $\mathrm{CU}<50 \%$ & $-15.9 \%$ & $83 \%$ & $-52.0 \%$ \\
\hline $31-60$ & $\begin{array}{c}50 \%<\mathrm{CU}< \\
75 \%\end{array}$ & $-13.6 \%$ & $87 \%$ & $-43.4 \%$ \\
\hline $61-90$ & $\begin{array}{c}75 \%<\mathrm{CU} \\
100 \%\end{array}$ & $-4.9 \%$ & $63 \%$ & $-41.4 \%$ \\
\hline $91-120$ & $\mathrm{CU}>87 \%$ & $-2.1 \%$ & $63 \%$ & $-42.5 \%$ \\
\hline $1-120$ & & $-9.1 \%$ & $74 \%$ & $-52.0 \%$ \\
\hline
\end{tabular}

Table 5 - Results with Modified Basic Period Independently Managed approach compared with Commons Cycle Jointly Managed approach over the 4 instances groups.

Looking at results reported in Table 5 it can be seen that, on the average, Modified BP IM outperforms CC JM in all instances groups, even if the average costs savings deteriorate as the capacity utilisation increases. On the average of all 120 instances, switching from CC JM to Modified BP IM guarantees an average cost saving of $9.1 \%$, and the amount of instances with cost saving is $74 \%$. The maximum cost saving reported in Tables 5 is considerable high and it ranges from a maximum of 52\% (for the instances 1-30 group) to a minimum of $41.4 \%$ (for the instances $61-90$ group). Then even if while the capacity utilisation increases the average costs savings deteriorate, there are instances in which Modified BP IM can guarantee a considerably high savings as showed for instance \#101 with CU of $95 \%$ and cost savings is close to $40 \%$. 
Finally we have to note that Modified BP IM presented in this paper can be more easily implemented, compared with JM CC proposed by Tang and Teunter (2006). The latter one is claimed to be "very time consuming for some examples with high capacity utilization, and therefore the algorithm needed to be terminated before an optimal solution was reached". Current proposed algorithms can be easily implemented in a spreadsheet (e.g. MS Excel and coded with some routines in VBA). In a laptop with a Centrino Processor of $2.13 \mathrm{GHz}$ and $2 \mathrm{~GB}$ RAM, even for the instances with the highest capacity utilization, it takes a maximum of 0.36, 2.19 and 29.40 seconds for CC IM, BP IM and Modified BP IM, respectively. The complete set of examples and results are available from the authors upon request.

\section{Conclusions}

The study proposed in this paper was motivated by the recent development of the ELSPR problem introduced by Tang and Teunter (2006) and Teunter et al (2009). In this paper, we propose a simplified approximation for the serviceable holding costs by splitting the $N$ items ELSPR into $2 \cdot N$ items ELSP. This approximation increases the serviceable holding cost, compared to the ELSPR modelled by Tang and Teunter (2006), but it provides an opportunity of relaxing the common cycle restriction for the solution generation. Thus, the increased cost in the approximation can be largely compensated by adopting more advanced scheduling policy such as the basic period (BP) policy. Moreover the basic period policy based algorithm proposed results in cost savings and it can be implemented in shorter computational time compared to already published results.

By numerically comparing the results of common cycle policy (Teunter et al, 2009) and the modified basic period policy proposed in this study in the 120 instances (according to the set of experiments designed by Teunter et al (2009)), we observe on the average a cost saving of about $9.1 \%$ and a maximum cost saving $52 \%$, with the $74 \%$ of the total instances with savings. Apart from the potential cost savings, an additional advantage is due to the short computational time, since our approach can be easily implemented in a spreadsheet package.

A possible direct development is to extend the current basic period policy to the case with TimeVarying Lot-Size policy proposed by Dobson (1987) or the further heuristics development by Raza and Akgunduz (2008). The cost savings can be expected, but the algorithm and computation time may also increase substantially. Moreover it can be possible to consider 2 separate production lines for manufactured and remanufactured item lots, as it has been already proposed by Teunter et al (2008). 


\section{Acknowledgements}

This work is a part of the INTERLINK project on Supply Chain Sustainability and it is supported by the Italian Ministry of Research and Higher Education. Participating universities are University of Brescia (Italy), Linköping Institute of Technology and Luleå University of Technology (both Sweden), University of Mannheim and University of Magdeburg (both Germany).

\section{References}

Abdul-Jalbar B., Segerstedt A., Sicilia J., Nilsson A. (2010). A new heuristic to solve the onewarehouse N-retailer problem, Computers \& Operations Research, 37(2), 265-272.

Bomberger E. E. (1966). A dynamic programming approach to a lot size scheduling problem, Management Science, 12(11), 778-784.

Cooke D.L., Rohleder T.R., Silver E.A. (2004). Finding effective schedules for the economic lot scheduling problem: A simple mixed integer programming approach, International Journal of Production Research, 42(1), 21-36.

Davis S. G. (1990). Scheduling economic lot size production runs, Management Science, 36(8), 985998.

Dobson G. (1987). The economic lot-scheduling problem: achieving feasibility using time-varying lot sizes, Operations Research, 35(5), 764-771.

Doll C. L., Whybark D. C. (1973). An iterative procedure for the single-machine multi-product lot scheduling problem, Management Science, 20(1), 50-55.

Elmaghraby S. E. (1978). The economic lot scheduling problem (ELSP): review and extensions, Management Science, 24(6), 587-598.

Hsu W-L. (1983). On the general feasibility test of scheduling lot sizes for several products on one machine, Management Science, 29(1), 93-105.

Levén E., Segerstedt A. (2007). A scheduling policy for adjusting economic lot quantities to a feasible solution, European Journal of Operational Research, 179(2), 414-423. 
Nilsson A, Segerstedt A, van der Sluis E. (2007). A new iterative heuristic to solve the joint replenishment problem using a spread-sheet technique, International Journal of Production Economics, 108(1-2), 399-405.

Nilsson K., Segerstedt, A. (2008). Corrections of costs to feasible solutions of economic lot scheduling problems, Computers and Industrial Engineering, 54(1), 155-168.

Raza, A.S., Akgunduz, A. (2008). A comparative study of heuristic algorithms on Economic Lot Scheduling Problem ,Computers and Industrial Engineering, 55(1), 94-109.

Rogers J. (1958). A computational approach to the economic lot scheduling problem, Management Science, 4(3), 264-291.

Roundy R. (1989). Rounding off to powers of two in continuous relaxations of capacitated lot sizing problems, Management Science, 35(12),1433-1442.

Segerstedt A. (1999). Lot sizes in a capacity constrained facility with available initial inventories, International Journal of Production Economics, 59(1-3), 469-475.

Tang O., Teunter R.H. (2006). Economic lot scheduling problem with returns, Production and Operations Management, 15(4), 488-497.

Teunter R., Kaparis K., Tang O. (2008). Multi-product economic lot scheduling problem with separate production lines for manufacturing and remanufacturing, European Journal of Operational Research, 191(3), 1241-1253.

Teunter R., Tang O., Kaparis K. (2009). Heuristics for the economic lot scheduling problem with returns, International Journal of Production Economics, 118(1), 323-330.

Tunasar C., Rajgopal, J. (1996). An evolutionary computation approach to the economic lot scheduling problem, Technical Report No.TR 96-1, Department of Industrial Engineering, University of Pittsburgh. 